\title{
Standart Olmayan Tipten İnterval Değerli Fuzzy Sayıların Dizi Uzayları Üzerine
}

\author{
Zarife ZARARSIZ1,*, Mehmet ŞENGÖNÜL ${ }^{1}$ \\ ${ }^{1}$ Nevşehir Üniversitesi, Fen Edebiyat Fakültesi, Matematik Bölümü, Nevşehir
}

Özet

Bu çalışmada $Z^{p}=\left(z_{n k}\right)^{p}$ p. Dereceden Zweier matrisi olmak üzere $c_{0}\left(E^{2}, Z^{P}\right), c\left(E^{2}, Z^{P}\right)$ ve $l_{\infty}\left(E^{2}, Z^{P}\right)$ ile gösterilen interval değerli bulanık sayıların sırasıyla Zweier sıfıra yakınsak, Zweier yakınsak ve Zweier sınırlı dizi kümeleri tanımlanarak bu kümelerin topolojik ve kapsama gibi önemli özellikleri ele alındı.

Anahtar Kelimeler: İnterval değerli fuzzy sayı, fuzzy küme, fuzzy sayı, matris dönüşümü, zweier matrisi.

\section{On the Sequence Spaces of Interval Valued Fuzzy Numbers which are Nonstandart}

\begin{abstract}
In this article some topological and algebraic properties of spaces of $c_{0}\left(E^{2}\right), c\left(E^{2}\right)$ and $l_{\infty}\left(E^{2}\right)$ which are convergent to $\theta$, convergent and bounded sequence spaces of interval valued fuzzy numbers are given, respectively. After that by taking a nonnegative, regular Zweier matrix $Z=\left(z_{n k}\right)$ and $\lambda\left(E^{2}\right) \in\left\{l_{\infty}\left(E^{2}\right), c_{0}\left(E^{2}\right), c\left(E^{2}\right)\right\}$, we have defined the sequence spaces of $c_{0}\left(E^{2}, Z^{P}\right), c\left(E^{2}, Z^{P}\right)$ ve $l_{\infty}\left(E^{2}, Z^{P}\right)$ called Zweier null, Zweier convergent and Zweier bounded sequence sets of interval valued fuzzy numbers, respectively. Finally some topological and inclusion problems on these spaces are given.
\end{abstract}

Keywords: Interval valued fuzzy number, fuzzy set, fuzzy number, matrix transformation, zweier matrix.

*e-mail: zarifezararsiz@nevsehir.edu.tr 


\section{Giriş}

İki değerli mantık sistemi ve bu mantık sisteminin ortaya koyduğu matematiksel yapılar, son zamanlarda bulanık mantık sistemi ve bu sistemin ortaya koyduğu esnek yaklaşım metodolojisi ile ortaya konan genel matematiksel yapılara yerini bırakmaya başlamıştır. Zadeh'in başlattığı bulanık mantık ve bulanık küme çalışmalarını, Matloka'nın [1] bulanık sayıların sınırlı ve yakınsak dizi tanımlarını vermesi; bulanık küme fikrini dizi uzayları ve

toplanabilme teorisine taşımıştır. Nanda [2], Matloka'nın çalışmalarını referans alarak bulanık sayıların sınırlı ve yakınsak dizilerinin tam metrik uzay olduğunu göstermiştir. Bir kaç yıl önce Talo ve Başar [3] bazı bulanık sayı dizilerinin kümelerinin duallerini belirleyip matris dönüşümleri hakkında önemli teoremler vermişlerdir. Hong [4] ise bulanık sayıların çekirdeğini incelemiştir. Bulanık kümelerin iyi bilinen bir genellemesi olan interval değerli bulanık küme fikri Gorzalczany [5] ve Turksen [6] tarafindan ortaya konmuştur. Son zamanlarda Chen [7] interval

değerli bulanık kümeler arasındaki uzaklığı, Guijun ve Xiaoping [8] de interval değerli bulanık sayıları tanımlamıştır. Meenakshi ve Kaliraja [9] da interval değerli bulanık sayıların değişik özellikleriyle ilgili çalışmalar yapmışlardır. Şengönül ve Zararsız [10] da bulanık sayıların yakınsak ve sınırlı dizi uzayları hakkında çalışmalar yapmışlardır.

Bu çalışmada, yukarıda sözü edilen çalışmların bir devamı olarak, interval değerli bulanık sayıların Zweier sınırlı, Zweier yakınsak ve Zweier null dizilerinin uzayları inşa edilerek bazı özellikleri araştırılmıştır.

\section{Temel Tanım ve Notasyonlar}

$X$, genel elemanı $x$ ile gösterilen boştan farklı bir küme olsun. $X^{\prime}$ 'in bir $A$ bulanık alt kümesi, $X^{\prime}$ 'in herbir elemanını $[0,1]$ intervaline ait bir reel sayıya karşılıkgetiren $\mu_{A}$ fonksiyonu ile karakterize edilir, [11]. $\mu_{A}$ üyelik fonksiyonu

$$
\mu_{A}: \mathrm{X} \rightarrow[0,1]
$$

şeklinde tanımlandığından $X^{\prime}$ in bir $A$ bulanık alt kümesi $A=\left\{\left(x, \mu_{A}(x)\right): x \in X\right\}$ biçiminde yazılabilir. Genel olarak $\mu_{A}$ fonksiyonu $x \in X^{\prime}$ in üyelik fonksiyonu olarak isimlendirilir. Bir bulanık $A$ kümesini $\alpha$ kesim kümeleri yardımıyla iç içe geçmiş intervallerin ailesi olarak düşünülebiliriz. Örneğin, B.S. Butkiewicz [12] bu bağıntıyı Fourier dönüşümlerini intervallere ve bulanık sayılara genişletmek için kullanmıştır.

Yukarıda açıkladığımız gibi; bir $X$ evrensel kümesinin bir $A$ bulanık alt kümesini belirlerken, $A$ kümesini oluşturan elemanların görüntüleri $[0,1]$ aralığındaki bir reel sayı ile belirlenir. Bulanık kümelerin belirlenmesinde kullandığımız üyelik fonksiyonları $X$ de alınan her elemana [0,1] aralığında kesin ve tek bir değer karşıllk getirir. Ancak üyelik derecelerini kesin bir değerle belirlemenin mümkün olmadığı durumlarda, bu elemanlara değer kümesinde üyeliğin derecesi olarak [0,1] aralığının bir alt aralığını karşılık getirmek fikrinin işleri çok daha kolaylaştıracağı görülmüştür, [13]. Böylece bulanık kümelerin bir genişletmesi olan ve interval değerli bulanık küme olarak adlandırılan yeni bir küme tanımı verilmiştir, $[6,11] . I=[0,1]$ intervalinin kapalı alt intervallerinin kümesi $[I]$ ve $X$ bir evrensel küme 
olmak üzere $u: X \rightarrow[I], x \rightarrow u(x)$ fonksiyonuna veya başka bir ifade ile $A=\left\{\left(x,\left[\alpha_{1}, \alpha_{2}\right]\right): x \in X, 0 \leq\right.$ $\left.\alpha_{1} \leq \alpha_{2} \leq 1\right\}$ kümesine $X$ üzerinde interval değerli bulanık küme denir, [6]. $\forall x \in X$ için $\alpha_{1}=\alpha_{2}$ olduğunda interval değerli bulanık küme bildiğimiz klâsik bulanık kümeye dönüşür. İnterval değerli bulanık kümelerde bir elemanın kümeye üyeliğinin derecesi, bulanık kümeler de olduğu gibi kesin değildir. Bir $X$ cümlesi üzerindeki bütün interval değerli bulanık kümelerin cümlesini $F^{2}(X)$ ile gösterelim. $u \in F^{2}(X)$ elemanı için $u^{-}(x)=\alpha_{1} \leq \alpha_{2}=u^{+}(x)$ olduğundan, $E^{1}$ üzerindeki kısmi stralama göz önünde tutulursa, $u(x)=\left[u^{-}(x), u^{+}(x)\right]$ yazılabilir.

Tanım 2.1. $u: R \rightarrow[I]$ fonksiyonu aşağıdaki şartları sağlıyorsa $u$ 'ya interval değerli bulanık sayı denir, [14].

(1) $u$ fonksiyonu normaldir. Yani en az bir $u_{0} \in R$ vardır öyleki $u\left(x_{0}\right)=\left[u^{-}\left(x_{0}\right), u^{+}\left(x_{0}\right)\right]=[1,1]$ dir.

(2) $u$ fonksiyonu bulanık konvekstir, yani $\forall x, y \in R$ ve $\mu \in[0,1]$ için $u[\mu x+(1-\mu) y] \geq$ $\min \{u(x), u(y)\}$ dir.

(3) $u^{-}$ve $u^{+}$üstten yarı süreklidir.

(4) $\left\{x \in R: u^{-}(x)>0, u^{+}(x)>0\right\}$ kümesinin kapanışı kompakttır.

İnterval değerli bulanık sayılarının kümesini $E^{2}$ ile göstereceğiz. Her bir $u \in E^{2}$ için $u(x)=$ $\left[u^{-}(x), u^{+}(x)\right], u^{-}(x) \leq u^{+}(x)$ ve $x \in R$ dir ve $u^{-}(x): R \rightarrow I$ ve $u^{+}(x): R \rightarrow I, R$ üzerinde iki bulanık sayıyı göstermektedir. $E^{2}$ üzerindeki kısmi sıralama bağıntısı $u \leq v \Leftrightarrow\left[u^{-}, u^{+}\right] \leq\left[v^{-}, v^{+}\right] \Leftrightarrow u^{-} \leq$ $v^{-}$ve $u^{+} \leq v^{+}$ile verilir.

Teorem 2.1. Bütün bulanık sayıların kümesi $E^{1}$, interval değerli bulanık sayılarının kümesi, $E^{2}$ 'nin içine gömülebilir, [15].

$u, v \in E^{2}$ olmak üzere iki interval değerli bulanık sayı arasındaki uzaklık;

$$
\overline{\bar{D}}(u, v)=\max \left\{\sup _{\alpha \in[0,1]} d\left(u^{-\alpha}, v^{-\alpha}\right), \sup _{\alpha \in[0,1]} d\left(u^{+\alpha}, v^{+\alpha}\right)\right\}
$$

ile verilir, [14].

Lemma 2.1. Bütün interval değerli bulanık sayıların kümesi $E^{2},(2.1)$ de verilen metrikle beraber bir metrik uzaydır, [14].

$u, v \in E^{2}$ ve $\lambda \in R$ olmak üzere iki interval değerli bulanık sayının toplamı, $u+v=\left[u^{-}(x), u^{+}(x)\right]+$ $\left[v^{-}(x), v^{+}(x)\right]=\left[u^{-\alpha}+v^{-\alpha}, u^{+\alpha}+v^{+\alpha}\right] \quad$ çarpımı, $\quad u . v=\left[u^{-\alpha}, u^{+\alpha}\right]\left[v^{-\alpha}, v^{+\alpha}\right]=$ $\left[\min \left\{u^{-\alpha} v^{-\alpha}, u^{-\alpha} v^{+\alpha}, u^{+\alpha} v^{-\alpha}, u^{+\alpha} v^{+\alpha}\right\}, \max \left\{u^{-\alpha} v^{-\alpha}, u^{-\alpha} v^{+\alpha}, u^{+\alpha} v^{-\alpha}, u^{+\alpha} v^{+\alpha}\right\}\right]$ ve skaler çarpım,

(1) $\lambda \geq 0$ için $u=\left[u^{-}(x), u^{+}(x)\right] \Rightarrow \lambda u=\left[\lambda u^{-}(x), \lambda u^{+}(x)\right]$

(2) $\lambda<0$ için $u=\left[u^{-}(x), u^{+}(x)\right] \Rightarrow \lambda u=\left[\lambda u^{+}(x), \lambda u^{-}(x)\right]$

olarak tanımlanır. 
$w\left(E^{2}\right)=\left\{\left(u_{k}\right):\left(\left[u_{k}{ }^{-}, u_{k}{ }^{+}\right]\right): u: N \rightarrow E^{2}, k \rightarrow u(k)=\left[u_{k}{ }^{-}, u_{k}{ }^{+}\right]\right.$ve $\left.u_{k}{ }^{-}, u_{k}{ }^{+} \in E^{1}\right\}$ kümesine interval değerli bulanık sayıların dizilerinin kümesi denir, [15].

$u=\left(u_{k}\right) \in w\left(E^{2}\right)$ olsun. $u$ dizisinin sınırlı olması için gerek ve yeter şart $\widetilde{m}, \widetilde{M} \in E^{2}$ ve $\forall k \in N$ için $\widetilde{m} \leq u_{k} \leq \widetilde{M}$ olmasidir, [15].

$u=\left(u_{k}\right)$ interval değerli bulanık sayı dizisi, $u_{0} \in E^{2 \prime}$ ye yakınsaktır $\Leftrightarrow \forall \varepsilon>0$ için $m$ pozitif tamsayısı vardır öyleki $\forall k \geq m$ için $\widetilde{D}\left(u_{k}, u_{0}\right)<\varepsilon$ ise. Eğer bu limit mevcutsa kısaca, $\lim _{k} u_{k}=u_{0}$, şeklinde gösterilir. Başka bir ifade ile eğer $\forall \varepsilon>0$ için $k \geq m$ olacak şekilde $m \in N$ mevcut öyleki

$$
\widetilde{D}\left(u_{k}, u_{0}\right)=\sup _{k \in N} \max \left\{\bar{d}\left(u_{k}{ }^{-}, v_{k}{ }^{-}\right), \bar{d}\left(u_{k}{ }^{+}, v_{k}{ }^{+}\right)\right\}<\varepsilon
$$

ise interval değerli fuzzy sayıların dizisi $\left(u_{k}\right), u_{0}{ }^{\prime}$ a yakınsaktır denir, [14]. İnterval değerli bulanık sayılarının $u$ dizisine Cauchy dizisi denir $\Leftrightarrow \forall \varepsilon>0$ ve $i, j>k$ olacak şekildeki $i, j$ pozitif tamsayıları için $\widetilde{D}\left(u_{i}, u_{j}\right)<\varepsilon$ ise, [11].

İnterval değerli bulanık sayıların, yakınsak, sıfıra yakınsak ve sınırlı dizilerinin uzayları, sırasıyla $c\left(E^{2}\right), c_{0}\left(E^{2}\right)$ ve $l_{\infty}\left(E^{2}\right)$ ile gösterilen, aşağıdaki biçimde tanımlı kümelerdir:

$$
\begin{aligned}
& c\left(E^{2}\right)=\left\{u \in w\left(E^{2}\right): \lim _{k} \max \left\{\bar{d}\left(u_{k}{ }^{-}, u_{0}{ }^{-}\right), \bar{d}\left(u_{k}{ }^{+}, u_{0}{ }^{+}\right)\right\}=0\right\}, \\
& c_{0}\left(E^{2}\right)=\left\{u \in w\left(E^{2}\right): \lim _{k} \max \left\{\bar{d}\left(u_{k}{ }^{-}, \theta^{-}\right), \bar{d}\left(u_{k}{ }^{+}, \theta^{+}\right)\right\}=0\right\}, \\
& l_{\infty}\left(E^{2}\right)=\left\{u \in w\left(E^{2}\right): \sup _{k} \max \left\{\bar{d}\left(u_{k}{ }^{-}, \theta^{-}\right), \bar{d}\left(u_{k}{ }^{+}, \theta^{+}\right)\right\}<\infty\right\} .
\end{aligned}
$$

Aşağıdaki teorem yukarıda tanımlanan kümelerin $w\left(E^{2}\right)$ içinde birbirine göre konumlarını göstermesi açısından ilginçtir.

Teorem 2.2. $c_{0}\left(E^{2}\right) \subset c\left(E^{2}\right) \subset l_{\infty}\left(E^{2}\right)$ kapsamaları mevcuttur, [15].

Teorem 2.3. Sıfıra yakınsak bulanık sayıların kümesi $c_{0}\left(E^{1}\right)$, yakınsak bulanık sayıların kümesi $c\left(E^{1}\right)$ ve sınırlı bulanık sayıların kümesi $l_{\infty}\left(E^{1}\right)$ sırasıyla $c_{0}\left(E^{2}\right), c\left(E^{2}\right)$ ve $l_{\infty}\left(E^{2}\right)$ kümeleri içine gömülebilir, [15].

\section{Esas Sonuçlar}

$\lambda\left(E^{2}\right)$ ve $\mu\left(E^{2}\right)$ interval değerli bulanık sayıların iki dizi uzayı ve $A=\left(a_{n k}\right)$ da reel sayıların sonsuz matrisi olsun, $(n, k \in N)$. Eğer her bir $u=\left(u_{k}\right) \in \lambda\left(E^{2}\right)$ için $u$ 'nun $A$ altındaki resmi, $A u, \mu\left(E^{2}\right)$, nin elemanı ise $A^{\prime}$ ya $\lambda\left(E^{2}\right)$ den $\mu\left(E^{2}\right)^{\prime}$ ye bir matris dönüşümü denir. İnterval değerli bulanık sayıların $\lambda\left(E^{2}\right)$ dizi uzayı verilsin.

$$
\lambda_{A}\left(E^{2}\right)=\left\{u=\left(u_{k}\right) \in w\left(E^{2}\right): A u \in \lambda\left(E^{2}\right)\right\}
$$


ile tanımlı $\lambda_{A}\left(E^{2}\right)$ kümesi, $A$ matrisinin etki alanı olarak adlandırılır. $p \neq 1$ olmak üzere $Z^{p}$,

$$
Z^{p}=\left(Z_{n k}\right)^{p}=\left\{\begin{array}{c}
p, n=k \text { ise } \\
1-p, n-1=k \text { ise }(n, k \in N) \\
0, \text { diğer durumlarda }
\end{array}\right.
$$

şeklinde tanımlanan sonsuz matrise genel Zweier matrisi denir. Eğer $A=Z$ olarak alınırsa (3.1) ile tanımlı küme genel Zweier matrisinin etki alanı olarak adlandırılır. Zweier yakınsak interval değerli bulanık sayıların yakınsak, sıfıra yakınsak ve sınırlı dizi kümeleri sırasıyla,

$$
\begin{gathered}
c\left(E^{2}, Z^{p}\right)=\left\{u=\left(u_{k}\right) \in w\left(E^{2}\right):\left(Z^{p} u\right) \in c\left(E^{2}\right)\right\}, \\
c_{0}\left(E^{2}, Z^{p}\right)=\left\{u=\left(u_{k}\right) \in w\left(E^{2}\right):\left(Z^{p} u\right) \in c_{0}\left(E^{2}\right)\right\}, \\
l_{\infty}\left(E^{2}, Z^{p}\right)=\left\{u=\left(u_{k}\right) \in w\left(E^{2}\right):\left(Z^{p} u\right) \in l_{\infty}\left(E^{2}\right)\right\}
\end{gathered}
$$

ile tanımlanır. Çalışmamızın bundan sonraki kısmında $p=\frac{1}{2}$ alınacaktır. $u=\left(u_{i}\right)$ dizisinin $Z^{\frac{1}{2}}$ dönüşümü $v=\left(v_{i}\right)$ olsun, yani

$$
\left(Z^{\frac{1}{2}} \mathrm{u}\right)_{i}=v_{i}=\frac{1}{2} u_{i}+\frac{1}{2} u_{i-1}=\frac{1}{2}\left(u_{i}+u_{i-1}\right) \text { olsun. }
$$

Teorem 3.1. $c\left(E^{2}, Z^{\frac{1}{2}}\right), c_{0}\left(E^{2}, Z^{\frac{1}{2}}\right)$ ve $l_{\infty}\left(E^{2}, Z^{\frac{1}{2}}\right)$ uzayları sirasiyla $c\left(E^{2}\right), c_{0}\left(E^{2}\right)$ ve $l_{\infty}\left(E^{2}\right)$ uzaylarına lineer olarak izomorfiktir. Yani;

$$
c\left(E^{2}, Z^{\frac{1}{2}}\right) \cong c\left(E^{2}\right), c_{0}\left(E^{2}, Z^{\frac{1}{2}}\right) \cong c_{0}\left(E^{2}\right) \text { ve } l_{\infty}\left(E^{2}, Z^{\frac{1}{2}}\right) \cong l_{\infty}\left(E^{2}\right)
$$

dir.

İspat: Bunun için ilk olarak $l_{\infty}\left(E^{2}, Z^{\frac{1}{2}}\right)$ ve $l_{\infty}\left(E^{2}\right)$ uzayları arasında lineer, birebir, örten bir dönüşümün varlığını göstermeliyiz. Bu dönüşümü $T$ ile gösterelim, yani;

$$
T: l_{\infty}\left(E^{2}, Z^{\frac{1}{2}}\right) \rightarrow l_{\infty}\left(E^{2}\right), T u=z, z=\left(z_{i}\right), i \in N, z_{i}=\frac{1}{2}\left(u_{i}+u_{i-1}\right)=\frac{1}{2}\left(\left[u_{i}^{-}, u_{i}^{+}\right]+\left[u_{i-1}{ }^{-}, u_{i-1}{ }^{+}\right]\right)
$$

olsun. Öncelikle $u, v \in l_{\infty}\left(E^{2}, Z^{\frac{1}{2}}\right)$ olmak üzere;

$$
\begin{aligned}
& \text { (1) } T(u+v)=\frac{1}{2}\left[\left(u_{i}+v_{i}\right)+\left(u_{i-1}+v_{i-1}\right)\right]=\frac{1}{2}\left\{\left[u_{i}^{-}+v_{i}^{-}, u_{i}^{+}+v_{i}^{+}\right]+\left[u_{i-1}{ }^{-}+\right.\right. \\
& \left.\left.v_{i-1}{ }^{-}, u_{i-1}^{+}+v_{i-1}^{+}\right]\right\}=\frac{1}{2}\left\{\left[u_{i}^{-}+u_{i-1}^{-}, u_{i}^{+}+u_{i-1}^{+}\right]+\left[v_{i}^{-}+v_{i-1}{ }^{-}, v_{i}^{+}+v_{i-1}^{+}\right]\right\}= \\
& \quad \frac{1}{2}\left(u_{i}+u_{i-1}\right)+\frac{1}{2}\left(v_{i}+v_{i-1}\right) \\
& =T u+T v,
\end{aligned}
$$

(2) Eğer $\alpha \in R$ ise $T\left(\alpha\left[u_{i}^{-}, u_{i}^{+}\right]\right)=T\left(\left[\alpha u_{i}{ }^{-}, \alpha u_{i}{ }^{+}\right]\right)=\frac{1}{2}\left(\left[\alpha u_{i}{ }^{-}, \alpha u_{i}{ }^{+}\right]+\left[\alpha u_{i-1}{ }^{-}, \alpha u_{i-1}{ }^{+}\right]\right)=\alpha T u$ dir. 
(1) ve (2) den $T$ dönüşümü lineerdir.

$T$ bire birdir. Gerçekten $T: l_{\infty}\left(E^{2}, Z^{\frac{1}{2}}\right) \rightarrow l_{\infty}\left(E^{2}\right), T u=v$ den $T\left(u_{i}\right)=T\left(v_{i}\right) \Rightarrow T\left(u_{i}\right)=\frac{1}{2} u_{i}+$ $\frac{1}{2} u_{i-1}, T\left(v_{i}\right)=\frac{1}{2} v_{i}+\frac{1}{2} v_{i-1} \quad$ dir. $\quad T\left(u_{i}\right)=T\left(v_{i}\right) \Rightarrow \frac{1}{2} u_{i}+\frac{1}{2} u_{i-1}=\frac{1}{2} v_{i}+\frac{1}{2} v_{i-1} \quad$ eşitliği $\quad i=0 \quad$ için $\frac{1}{2} u_{0}=\frac{1}{2} v_{0} \Rightarrow u_{0}=v_{0}, i=1$ için $\frac{1}{2} u_{1}+\frac{1}{2} u_{0}=\frac{1}{2} v_{1}+\frac{1}{2} v_{0} \Rightarrow u_{1}=v_{1}$ dir. $i=r$ için doğru olduğunu kabul edelim. Yani $u_{r}=v_{r}$ olsun. Buradan $\frac{1}{2} u_{r+1}+\frac{1}{2} u_{r}=\frac{1}{2} v_{r+1}+\frac{1}{2} v_{r} \Rightarrow u_{r+1}=v_{r+1}$ olduğundan tümevarım prensibi gereğince $\forall i$ için $u_{i}=v_{i}$ olur. $\mathrm{O}$ halde $T$ birebirdir. Şimdi $v \in l_{\infty}\left(E^{2}\right)$ için $u_{i}=$ $2 \sum_{j=0}^{i}(-1)^{i-j} v_{j},(i \in N)$ dizisini göz önüne alalım.

$\lim _{i \rightarrow \infty} \frac{1}{2}\left(u_{i}+u_{i-1}\right)=\frac{1}{2} \lim _{i \rightarrow \infty} 2 \sum_{j=0}^{i}(-1)^{i-j} v_{j}+\frac{1}{2} \lim _{i \rightarrow \infty} 2 \sum_{j=0}^{i-1}(-1)^{i-1-j} v_{j}=\lim _{i \rightarrow \infty} v_{i} \Rightarrow u \in l_{\infty}\left(E^{2}, Z^{\frac{1}{2}}\right)$, yani $T$ örtendir. Şu halde $l_{\infty}\left(E^{2}, Z^{\frac{1}{2}}\right) \cong l_{\infty}\left(E^{2}\right)$ dir.

Benzer olarak $c\left(E^{2}, Z^{\frac{1}{2}}\right) \cong c\left(E^{2}\right), c_{0}\left(E^{2}, Z^{\frac{1}{2}}\right) \cong c_{0}\left(E^{2}\right)$ olduğu görülür.

Teorem 3.2. $u_{n}$ bulanık sayıların bir dizisi olsun. Eğer $n \rightarrow \infty$ için $D\left(u_{n}, u_{0}\right) \rightarrow 0$ ise o zaman $n \rightarrow \infty$ için

$D\left(p u_{n}+(1-p) u_{n-1}, u_{0}\right) \rightarrow 0$ dır. Diğer bir söyleyişle $Z^{\frac{1}{2}}$ Zweier matrisi regülerdir, [15].

İspat: Bulanık sayıların $\left(u_{n}\right)$ dizisi $u_{0}$ bulanık sayısına yakınsak olsun. $\mathrm{O}$ zaman $\forall \varepsilon>0$ için bir $n_{0}$ pozitif tamsayısı vardır öyleki $\forall n \geq n_{0}$ için $D\left(u_{n}, u_{0}\right)<\frac{\varepsilon}{2 M}$ olur. $D\left(Z^{\frac{1}{2}} u, u_{0}\right)=D\left(p u_{n}+(1-\right.$ $\left.p) u_{n-1}, u_{0}\right) \leq p D\left(u_{n}, u_{0}\right)+(1-p) D\left(u_{n-1}, u_{0}\right)$ yazılabilir. $M=\max \{p,(1-p)\}$ olarak seçilirse $D\left(Z^{\frac{1}{2}} u, u_{0}\right)<\varepsilon$ olur. Yani $\lim _{n}\left(p u_{n}+(1-p) u_{n-1}\right)=u_{0}$ eşitliği sağlanır. $\square$

Teorem 3.3. $c\left(E^{2}, Z^{\frac{1}{2}}\right), c_{0}\left(E^{2}, Z^{\frac{1}{2}}\right)$ ve $l_{\infty}\left(E^{2}, Z^{\frac{1}{2}}\right)$ uzayları arasinda $c_{0}\left(E^{2}, Z^{\frac{1}{2}}\right) \subset c\left(E^{2}, Z^{\frac{1}{2}}\right) \subset$ $l_{\infty}\left(E^{2}, Z^{\frac{1}{2}}\right)$ kapsamaları geçerlidir.

İspat: $c_{0}\left(E^{2}, Z^{\frac{1}{2}}\right) \subset c\left(E^{2}, Z^{\frac{1}{2}}\right)$ olduğu açıktır. $c\left(E^{2}, Z^{\frac{1}{2}}\right) \subset l_{\infty}\left(E^{2}, Z^{\frac{1}{2}}\right)$ olduğunu gösterelim: $u_{k} \in$ $c\left(E^{2}, Z^{\frac{1}{2}}\right)$ olsun. Bu $\lim _{k \rightarrow \infty} \max \left\{\bar{d}\left(Z^{\frac{1}{2}} u_{k}{ }^{-}, u_{0}{ }^{-}\right), \bar{d}\left(Z^{\frac{1}{2}} u_{k}{ }^{+}, u_{0}{ }^{+}\right)\right\}=0 \quad$ demek olduğundan $\bar{d}\left(Z^{\frac{1}{2}} u_{k}{ }^{-}, u_{0}{ }^{-}\right)<\varepsilon$ ve $\bar{d}\left(Z^{\frac{1}{2}} u_{k}{ }^{+}, u_{0}{ }^{+}\right)<\varepsilon$ yazılabilir. Dolayıs ile $\left(Z^{\frac{1}{2}} u_{k}{ }^{-}\right) \in c\left(E^{1}, Z^{\frac{1}{2}}\right)$ ve $\left(Z^{\frac{1}{2}} u_{k}{ }^{+}\right) \in$ $c\left(E^{1}, Z^{\frac{1}{2}}\right)$ olup $c\left(E^{1}, Z^{\frac{1}{2}}\right) \subset l_{\infty}\left(E^{1}, Z^{\frac{1}{2}}\right)$ kapsaması mevcuttur. Şu halde $\left(Z^{\frac{1}{2}} u_{k}^{-}\right) \in l_{\infty}\left(E^{1}, Z^{\frac{1}{2}}\right)$ ve $\left(Z^{\frac{1}{2}} u_{k}^{+}\right) \in l_{\infty}\left(E^{1}, Z^{\frac{1}{2}}\right)$ yazılabilir. Demekki $\left(Z^{\frac{1}{2}} u_{k}\right) \in l_{\infty}\left(E^{2}, Z^{\frac{1}{2}}\right)$ dir. Bu ise $\left(u_{k}\right) \in l_{\infty}\left(E^{2}, Z^{\frac{1}{2}}\right)$ olmas1 demektir.

Şimdi bir $u=\left(u_{k}\right) \in l_{\infty}\left(E^{2}, Z^{\frac{1}{2}}\right)$ dizisini; eğer $k$ çift ise, 
$u_{k}=\left\{\begin{array}{lc}x-1, & x \in[1,2] \text { ise } \\ 3-x & { }_{x} x \in[1,2] \text { ise } \\ 0, & \text { diğer durumlar }\end{array}, \begin{cases}\frac{x}{2}, & x \in[0,2] \text { ise } \\ \frac{5-x}{3}, & x \in[2,5] \text { ise } \\ 0, & \text { diğer durumlar }\end{cases}\right.$

ve eğer $\mathrm{k}$ tek ise,

$u_{k}=\left\{\begin{array}{ccc}x+3, & x \in[-3,-2] \text { ise } \\ -1-x & , x \in[-2,-1] \text { ise } \\ 0, & \text { diğer durumlar }\end{array},\left\{\begin{array}{cc}\frac{x+5}{3}, & x \in[-5,-2] \text { ise } \\ \frac{-x}{2}, & x \in[-2,0] \text { ise } \\ 0, & \text { diğer durumlar }\end{array}\right.\right.$

biçiminde tanımlayalım. Açık olarak $\lim _{k} u_{k}$ mevcut değildir. $u=u_{k} \operatorname{dizisinin} \alpha$ - kesimleri

$$
\left\{\begin{array}{l}
u_{k}^{\alpha}=[[\alpha+1,3-\alpha],[2 \alpha, 5-3 \alpha]], k \text { çift ise }, \\
u_{k}^{\alpha}=[[\alpha+1,3-\alpha],[2 \alpha, 5-3 \alpha]], k \text { tek ise }
\end{array}\right.
$$

olduğundan $v_{k}=Z^{\frac{1}{2}}\left(u_{k}^{\alpha}\right)=\frac{1}{2}[[2 \alpha-2,2-2 \alpha],[5 \alpha-5,5-5 \alpha]]$ elde edilir. $\alpha=0$ için $v_{k}^{0}=$ $[[-2,2],[-5,5]]$ ve $\alpha=1$ için $v_{k}{ }^{1}=[[0,0],[0,0]]$ bulunur. $Z^{\frac{1}{2}}\left(u_{k}{ }^{\alpha}\right)$ ' ya karşıllı gelen üyelik fonksiyonu göz önünde tutulursa

$c\left(E^{2}, Z^{\frac{1}{2}}\right) \subset l_{\infty}\left(E^{2}, Z^{\frac{1}{2}}\right)$ olduğu görülür. $\square$

Teorem 3.4. $c\left(E^{2}\right) \subset c\left(E^{2}, Z^{\frac{1}{2}}\right)$ ve $c_{0}\left(E^{2}\right) \subset c_{0}\left(E^{2}, Z^{\frac{1}{2}}\right)$ kapsamaları sağlanır.

İspat: $c\left(E^{2}\right) \subset c\left(E^{2}, Z^{\frac{1}{2}}\right)$ olduğunu gösterelim. $x \in c\left(E^{2}\right)$ olsun. $Z^{\frac{1}{2}}$ 'nin regüler olmasından dolayı $Z^{\frac{1}{2}} x \in c\left(E^{2}, Z^{\frac{1}{2}}\right)$ yazabiliriz. Bu ise $x \in c\left(E^{2}, Z^{\frac{1}{2}}\right)$ olması demektir. Buradan $c\left(E^{2}\right) \subset c\left(E^{2}, Z^{\frac{1}{2}}\right)$ kapsaması elde edilir. $\square$

Aşağıda vereceğimiz son teorem $\lambda\left(E^{2}, Z^{\frac{1}{2}}\right) \in\left\{c\left(E^{2}, Z^{\frac{1}{2}}\right), c_{0}\left(E^{2}, Z^{\frac{1}{2}}\right), l_{\infty}\left(E^{2}, Z^{\frac{1}{2}}\right)\right\}$ olmak üzere $\lambda\left(E^{2}, Z^{\frac{1}{2}}\right)$ cümlesi üzerindeki metrik ve bu metriğe göre tamlık hakkında olacaktır. $u, v \in \lambda\left(E^{2}, Z^{\frac{1}{2}}\right)$ ise $u$ ile $v$ arasındaki uzaklık;

$$
\widetilde{D}_{Z^{\frac{1}{2}}}(u, v)=\sup _{k} \max \{U, V\}
$$

şeklinde tanımlanır.

Burada $U=\sup _{\alpha \in[0,1]} d\left(Z^{\frac{1}{2}} u_{k}^{-\alpha}, Z^{\frac{1}{2}} v_{k}^{-\alpha}\right)$ ve $V=\sup _{\alpha \in[0,1]} d\left(Z^{\frac{1}{2}} u_{k}^{+\alpha}, Z^{\frac{1}{2}} v_{k}^{+\alpha}\right) \operatorname{dir}$.

Önce bir lemma verelim. 
Lemma 3.1.[16]

$$
\begin{gathered}
l_{\infty}\left(E^{1}, Z^{p}\right)=\left\{u=\left(u_{k}\right) \in w\left(E^{1}\right):\left(Z^{p} u\right) \in l_{\infty}\left(E^{1}\right)\right\}, \\
c\left(E^{1}, Z^{p}\right)=\left\{u=\left(u_{k}\right) \in w\left(E^{1}\right):\left(Z^{p} u\right) \in c\left(E^{1}\right)\right\}, \\
c_{0}\left(E^{1}, Z^{p}\right)=\left\{u=\left(u_{k}\right) \in w\left(E^{1}\right):\left(Z^{p} u\right) \in c_{0}\left(E^{1}\right)\right\}
\end{gathered}
$$

ile verilen $l_{\infty}\left(E^{1}, Z^{p}\right), c\left(E^{1}, Z^{p}\right)$ ve $c_{0}\left(E^{1}, Z^{p}\right)$ kümeleri

$$
\|u\|_{l_{\infty}\left(E^{1}, Z^{\eta}\right)}=\|u\|_{c\left(E^{1}, Z^{\eta}\right)}=\|u\|_{c_{0}\left(E^{1}, Z^{\eta}\right)}=\left\|\left(Z^{\eta} u\right)\right\|_{c\left(E^{1}\right)}
$$

normuna göre tamdır, burada $\left\|\left(Z^{\eta} u\right)\right\|_{c\left(E^{1}\right)}=\sup _{k} \bar{d}\left(Z^{\eta} u_{k}, \overline{0}\right) \operatorname{dir}$.

Teorem 3.5. $\lambda\left(E^{2}, Z^{\frac{1}{2}}\right) \in\left\{c\left(E^{2}, Z^{\frac{1}{2}}\right), c_{0}\left(E^{2}, Z^{\frac{1}{2}}\right), l_{\infty}\left(E^{2}, Z^{\frac{1}{2}}\right)\right\}$ olmak üzere $\lambda\left(E^{2}, Z^{\frac{1}{2}}\right)$, (3.7) de verilen metrik ile beraber tam metrik uzaydir.

İspat: İspatları birbirine benzediğinden biz sadece $\left(l_{\infty}\left(E^{2}, Z^{\frac{1}{2}}\right), \widetilde{D}_{Z^{\frac{1}{2}}}\right)$ 'nin tam metrik uzay olduğunu göstereceğiz.

(1)

$$
\begin{aligned}
& \widetilde{D}_{Z^{\frac{1}{2}}}(u, v)=0 \Leftrightarrow u=v \text { şartının sağlandığ } \\
& \widetilde{D}_{Z^{\frac{1}{2}}}(u, v)=\sup _{k} \max \left\{\sup _{\alpha \in[0,1]} d\left(Z^{\frac{1}{2}} u_{k}^{-\alpha}, Z^{\frac{1}{2}} v_{k}^{-\alpha}\right), \sup _{\alpha \in[0,1]} d\left(Z^{\frac{1}{2}} u_{k}^{+\alpha}, Z^{\frac{1}{2}} v_{k}^{+\alpha}\right)\right\}=0 \Leftrightarrow \\
& \left|Z^{\frac{1}{2}} u_{l k}{ }^{-\alpha}-Z^{\frac{1}{2}} v_{l k}{ }^{-\alpha}\right|=0,\left|Z^{\frac{1}{2}} u_{r k}{ }^{-\alpha}-Z^{\frac{1}{2}} v_{r k}{ }^{-\alpha}\right|=0,\left|Z^{\frac{1}{2}} u_{l k}^{+\alpha}-Z^{\frac{1}{2}} v_{l k}{ }^{+\alpha}\right|=0, \mid Z^{\frac{1}{2}} u_{r k}^{+\alpha}- \\
& Z^{\frac{1}{2}} v_{r k}{ }^{+\alpha} \mid=0 \Leftrightarrow Z^{\frac{1}{2}} u_{l k}^{-\alpha}=Z^{\frac{1}{2}} v_{l k}^{-\alpha}, Z^{\frac{1}{2}} u_{r k}{ }^{-\alpha}=Z^{\frac{1}{2}} v_{r k}{ }^{-\alpha}, Z^{\frac{1}{2}} u_{l k}^{+\alpha}=Z^{\frac{1}{2}} v_{l k}^{+\alpha}, Z^{\frac{1}{2}} u_{r k}{ }^{+\alpha}= \\
& Z^{\frac{1}{2}} v_{r k}{ }^{+\alpha} \Leftrightarrow u_{k}{ }^{-\alpha}=v_{k}{ }^{-\alpha}, u_{k}{ }^{+\alpha}=v_{k}{ }^{+\alpha} \Leftrightarrow{u_{k}}^{-}=v_{k}{ }^{-}, u_{k}{ }^{+}=v_{k}{ }^{+} \Leftrightarrow u=v \text { olmasindan ve }
\end{aligned}
$$

$$
\widetilde{D}_{Z^{\frac{1}{2}}}(u, v)=\sup _{k} \max \left\{\sup _{\alpha \in[0,1]} d\left(Z^{\frac{1}{2}} u_{k}^{-\alpha}, Z^{\frac{1}{2}} v_{k}^{-\alpha}\right), \sup _{\alpha \in[0,1]} d\left(Z^{\frac{1}{2}} u_{k}^{+\alpha}, Z^{\frac{1}{2}} v_{k}^{+\alpha}\right)\right\}=
$$

$\widetilde{D}_{Z^{\frac{1}{2}}}(u, v)=\sup _{k} \max \left\{\sup _{\alpha \in[0,1]} d\left(Z^{\frac{1}{2}} v_{k}^{-\alpha}, Z^{\frac{1}{2}} u_{k}^{-\alpha}\right), \sup _{\alpha \in[0,1]} d\left(Z^{\frac{1}{2}} v_{k}^{+\alpha}, Z^{\frac{1}{2}} u_{k}^{+\alpha}\right)\right\}=\widetilde{D}_{Z^{\frac{1}{2}}}(v, u)$

olduğu mutlak değer tanımından açıktır.

(3) $\widetilde{D}_{Z^{\frac{1}{2}}}(u, v) \leq \widetilde{D}_{Z^{\frac{1}{2}}}(u, w)+\widetilde{D}_{Z^{\frac{1}{2}}}(w, v)$ eşitsizliğinin sağlandığını gösterelim.

$\widetilde{D}_{Z^{\frac{1}{2}}}(u, v)=\sup _{k} \max \left\{\sup _{\alpha \in[0,1]}\left\{\left|Z^{\frac{1}{2}} u_{l k}^{-\alpha}-Z^{\frac{1}{2}} v_{l k}^{-\alpha}+Z^{\frac{1}{2}} w_{l k}^{-\alpha}-Z^{\frac{1}{2}} w_{l k}^{-\alpha}\right|, \mid Z^{\frac{1}{2}} u_{r k}^{-\alpha}-Z^{\frac{1}{2}} v_{r k}^{-\alpha}+\right.\right.$

$\left.Z^{\frac{1}{2}}{W_{r k}}^{-\alpha}-Z^{\frac{1}{2}}{W_{r k}}^{-\alpha} \mid\right\}, \sup _{\alpha \in[0,1]}\left\{\left|Z^{\frac{1}{2}} u_{l k}^{+\alpha}-Z^{\frac{1}{2}} v_{l k}^{+\alpha}+Z^{\frac{1}{2}} w_{l k}^{+\alpha}-Z^{\frac{1}{2}} w_{l k}^{+\alpha}\right|, \mid Z^{\frac{1}{2}} u_{r k}^{+\alpha}-Z^{\frac{1}{2}} v_{r k}^{+\alpha}+\right.$

$\left.\left.Z^{\frac{1}{2}} W_{r k}+\alpha-Z^{\frac{1}{2}} W_{r k}+\alpha \mid\right\}\right\} \leq$ 


$$
\begin{aligned}
& \sup _{k} \max \left\{\operatorname { s u p } _ { \alpha \in [ 0 , 1 ] } \left\{\left|Z^{\frac{1}{2}} u_{l k}^{-\alpha}-Z^{\frac{1}{2}} W_{l k}^{-\alpha}\right|+\left|Z^{\frac{1}{2}}{W_{l k}}^{-\alpha}-Z^{\frac{1}{2}} v_{l k}^{-\alpha}\right|,\left|Z^{\frac{1}{2}} u_{r k}^{-\alpha}-Z^{\frac{1}{2}} W_{r k}{ }^{-\alpha}\right|+\right.\right. \\
& \left.\left.\left|Z^{\frac{1}{2}}{W_{r k}}^{-\alpha}-Z^{\frac{1}{2}} v_{r k}{ }^{-\alpha}\right|\right\}\right\},\left\{\sup _{\alpha \in[0,1]}\left|Z^{\frac{1}{2}} u_{l k}^{+\alpha}-Z^{\frac{1}{2}}{W_{l k}}^{+\alpha}\right|+\left|Z^{\frac{1}{2}}{W_{l k}}^{+\alpha}-Z^{\frac{1}{2}} v_{l k}^{+\alpha}\right|,\left|Z^{\frac{1}{2}} u_{r k}^{+\alpha}-Z^{\frac{1}{2}} W_{r k}^{+\alpha}\right|+\right. \\
& \left.\left|Z^{\frac{1}{2}} W_{r k}+\alpha-Z^{\frac{1}{2}} v_{r k}+\alpha\right|\right\} \leq
\end{aligned}
$$$$
\sup _{k} \max \left\{\sup _{\alpha \in[0,1]}\left\{\left|Z^{\frac{1}{2}} u_{l k}^{-\alpha}-Z^{\frac{1}{2}} W_{l k}^{-\alpha}\right|,\left|Z^{\frac{1}{2}} u_{r k}{ }^{-\alpha}-Z^{\frac{1}{2}} W_{r k}{ }^{-\alpha}\right|\right\}, \sup _{\alpha \in[0,1]}\left\{\mid Z^{\frac{1}{2}} u_{l k}^{+\alpha}-\right.\right.
$$$$
\left.\left.Z^{\frac{1}{2}} W_{l k}^{+\alpha}|,| Z^{\frac{1}{2}} u_{r k}^{+\alpha}-Z^{\frac{1}{2}} W_{r k}^{+\alpha} \mid\right\}\right\}+\sup _{k} \max \left\{\operatorname { s u p } _ { \alpha \in [ 0 , 1 ] } \left\{\left|Z^{\frac{1}{2}} W_{l k}^{-\alpha}-Z^{\frac{1}{2}} v_{l k}^{-\alpha}\right|, \mid Z^{\frac{1}{2}}{W_{r k}}^{-\alpha}-\right.\right.
$$$$
\left.\left.Z^{\frac{1}{2}} v_{r k}{ }^{-\alpha} \mid\right\}, \sup _{\alpha \in[0,1]}\left\{\left|Z^{\frac{1}{2}}{W_{l k}}^{+\alpha}-Z^{\frac{1}{2}} v_{l k}^{+\alpha}\right|,\left|Z^{\frac{1}{2}} W_{r k}^{+\alpha}-Z^{\frac{1}{2}} v_{r k}^{+\alpha}\right|\right\}\right\}=\widetilde{D}_{Z^{\frac{1}{2}}}(u, w)+\widetilde{D}_{Z^{\frac{1}{2}}}(w, v) \Leftrightarrow
$$

$\widetilde{D}_{Z^{\frac{1}{2}}}(u, v) \leq \widetilde{D}_{Z^{\frac{1}{2}}}(u, w)+\widetilde{D}_{Z^{\frac{1}{2}}}(w, v)$ dır. Böylece metrik şartları sağlanmış olur. Varsayalım ki $\left(u_{k}^{i}\right)=$ $\left(u_{0}{ }^{i}, u_{1}{ }^{i}, u_{2}{ }^{i}, \ldots\right)$ her bir $i \in N$ için $l_{\infty}\left(E^{2}, Z^{\frac{1}{2}}\right)$ de bir Cauchy dizisi olsun. O halde $\forall \varepsilon>0$ için en az bir $n_{0}$ doğal sayısı vardır öyleki her $i, j>n_{0}$ için $\widetilde{D}_{Z^{\frac{1}{2}}}\left(u_{k}{ }^{i}, u_{k}{ }^{j}\right)<\varepsilon$ dır. Yani

$$
\widetilde{D}_{Z^{\frac{1}{2}}}\left(u_{k}^{i}, u_{k}^{j}\right)=\sup _{k} \max \left\{\sup _{\alpha \in[0,1]} d\left(Z^{\frac{1}{2}} u_{k}^{-\alpha i}, Z^{\frac{1}{2}} v_{k}^{-\alpha j}\right), \sup _{\alpha \in[0,1]} d\left(Z^{\frac{1}{2}} u_{k}^{+\alpha i}, Z^{\frac{1}{2}} v_{k}^{+\alpha j}\right)\right\}<\varepsilon
$$

olur. Buradan;

$$
d\left(Z^{\frac{1}{2}} u_{k}^{-\alpha i}, Z^{\frac{1}{2}} v_{k}^{-\alpha j}\right)<\varepsilon \text { ve } d\left(Z^{\frac{1}{2}} u_{k}^{+\alpha i}, Z^{\frac{1}{2}} v_{k}^{+\alpha j}\right)<\varepsilon
$$

olduğu görülür. $Z^{\frac{1}{2}} u_{k}^{-\alpha i}$ ve $Z^{\frac{1}{2}} u_{k}^{+\alpha i}$ dizileri $c\left(E^{1}, Z^{\frac{1}{2}}\right)$ de birer Cauchy dizisidir. Lemma 3.1 den, $c\left(E^{1}, Z^{\frac{1}{2}}\right)$ tam metrik uzay olduğundan $\lim _{i} Z^{\frac{1}{2}} u_{k}{ }^{-\alpha i}=u_{0}{ }^{-}$ve $\lim _{i} Z^{\frac{1}{2}} u_{k}{ }^{+\alpha i}=u_{0}{ }^{+}$olacak şekilde $u_{0}{ }^{-}$ve $u_{0}{ }^{+}$limitleri mevcuttur. Her $i, j \geq n_{0}$ için

$$
\lim _{j \rightarrow \infty} \widetilde{D}_{Z^{\frac{1}{2}}}\left(u_{k}^{i}, u_{k}^{j}\right)=\widetilde{D}_{Z^{\frac{1}{2}}}\left(u_{k}^{i}, \lim _{j \rightarrow \infty} u_{k}^{j}\right)=\widetilde{D}_{Z^{\frac{1}{2}}}\left(u_{k}^{i}, u_{0}\right)<\varepsilon
$$

olduğundan $\lim _{i} u_{k}^{i}=u_{0}$ dir. Diğer taraftan $u_{0} \in l_{\infty}\left(E^{2}, Z^{\frac{1}{2}}\right)$ olduğu açıktır.

\section{Kaynaklar}

[1] Matloka M.," Sequence of fuzzy numbers", BUSEFAL 28, 28, 28-37, 1986.

[2] Nanda S.," On sequence spaces of fuzzy numbers”, Fuzzy Sets and Systems, 33, 123-126, 1989.

[3] TaloÖ., Başar, F., "Determination of the duals of classical sets of sequences of fuzzy numbers related matrix transformations", Computers and Mathematics with Applications, 58, 717-733, 2009.

[4] Hong D.H., Moon E.L., Kim J.D., “A note on the core of fuzzy numbers”, Fuzzy Sets and Systems, 98,331-335, 1998. 
[5] Gorzalczany, B., "Aproximate inference with interval valued fuzzy sets”, Proc. Polish Symp., On interval and Fuzzy Math., Poznan, Poland, 89-95, 1983.

[6] Turksen B., "Interval valued fuzzy sets based on normal forms", Fuzzy Sets and Systems, $20,191210,1986$.

[7] Chen Shi-Jay, Chen Shyi-Ming, "Handling information filtering problems based on interval valued fuzzy numbers", Journal of the Chinese Institute of Engineers, 29, No. 1, pp. 83-96, 2006.

[8] Guijun W. and Xiaoping L., "The applications of interval valued fuzzy numbers and intervaldistribution numbers", Fuzzy Sets and Systems, 98 (1998), 331-335.

[9] Meenakshi A.R., Kaliraja M.," Regular interval valued fuzzy matrices”, Advanced in Fuzzy Mathematics, 5, 7-15, 2010.

[10] Şengönül M., Zararsız Z., "Some additions to the fuzzy convergent and fuzzy bounded sequence spaces of fuzzy numbers", Hindawi Publishing Corporation Abstract and Applied Analysis, Volume 2011, Article ID: 837584, Doi:10.1155/2011/837584.

[11] Zadeh L. A., "Fuzzy Sets”, Information and Control., 8, 338-353, 1965.

[12] Interval Talks at the International Conference on Information Processing and Management of Uncertainty in Knowledge-Based Sytstems, IPMU' 2006, Paris, France, July 2-7, 2006.

[13] Klir G., and Yuan B.," Fuzzy Sets and Fuzzy Logic”, Prentice Hall PTR, New Jersey, 07458.

[14] Li C., "Distance between interval-valued fuzzy sets", The 28th North American Fuzzy Information Processing Society Annual Conference, Cincinnati, Ohio, USA, 2009.

[15] Şengönül M., “The interval valued fuzzy sequence spaces”, (under comminication).

[16] Şengönül M., “On the Zweier sequence spaces of fuzzy numbers”, (under comminication ). 